\title{
A Prospective, Randomized Controlled Study To Compare Changes In Endotracheal Tube Cuff Pressure During General Anaesthesia Using Nitrous Oxide Between Air And Distilled Water Cuff Inflation Using Pressure Gauge And Pressure Transducer.
}

\author{
Dr. Barkha Gadpale ${ }^{1}$, Dr. Rajendra ${ }^{2}$ D. Patel ${ }^{3}$, Dr. Nirav M. Kotak $^{4}$
}

\begin{abstract}
Summary: In this prospective, randomized controlled study, we studied endotracheal tube cuff pressure changes in 100 patients undergoing elective surgical procedures requiring general anaesthesia with oxygen and nitrous oxide. Devices named pressure gauge and pressure transducer are compared as well. The cuffs were inflated with either air or distilled water. Mean cuff pressure in air filled cuffs increased throughout the procedure, reaching $37.58 \pm 0.93 \mathrm{mmHg}$ compared with $23.66 \pm 0.94 \mathrm{mmHg}$ in water filled cuffs at the end of 2 hours. The rise in cuff pressure were significantly lower $(P<0.05)$ in water filled cuffs. The endotracheal tube cuff distended with distilled water, rise in cuff pressure during general anaesthesia with nitrous oxide is lower than that of an air filled cuff.
\end{abstract}

Keywords: Cuff pressure, Endotracheal tube, Pressure gauge, Pressure transducer.

\section{Introduction}

The use of air to inflate the ETT cuff is associated with a pressure on the lateral wall of the trachea.This pressure should be limited to a range of 15 to $45 \mathrm{mmHg}$. ${ }^{1}$ During general anaesthesia,the use of Nitrous Oxide and Oxygen can defeat this safety measure If room air is used to inflate the cuff, because Nitrous Oxide diffuses 34 times as much faster than Nitrogen diffuses out potentially resulting endotracheal tube cuff overdistention. ${ }^{2}$ This causes an increase in intracuff pressure and a resultant pressure to be exerted on the tracheal mucosa.The result is a possible tracheal mucosal injury and an increased chance of post-extubation morbidity.

A number of devices are available for measurement of cuff pressure. Pressure transducer, which is otherwise used to record the intra-arterial blood pressures. ${ }^{3,4}$ This is done by attaching the length of a pressure line by means of a three-way stopcock to the pilot of the endotracheal tube off. All this time, the transducer was to be kept at a level of the manubrium streni and zeroed before starting recording. The transducers gave a continuous recording of the pressures with respect to time.

Other methods that have been used are the use of an pressure gauge ${ }^{5}$, which has a scale of pressure range and a pointer.Our study is to compare Pressure changes inside Endotracheal tube cuff when it is inflated with Air and when it is inflated with Distilled water. There has also been an attempt to determine relation of this pressure to the development of complications in postoperative period.

\section{Materials And Methods}

After obtaining institutional ethics committee approval and informed patient consent, 100 patients of ASA physical status I and II, aged between 19-65 years of age were studied. These patients underwent elective surgeries requiring general anaesthesia with nitrous oxide. Randomization of patients was done into two groups(group A and group W) by SNOSE (sequentially numbered, opaque sealed, stapled envelopes) method.Patients consent was taken. After attaching monitors like pulse oximeter, cardioscope, noninvasive blood pressure monitor/sphygmomanometer\& Capnography, preoperative readings were noted. 18G IV angiocatheter was used to secure IV line. Patients were premedicated with Inj. Ramosetron $0.3 \mathrm{mg}$ IV \&Inj. Ranitidine $50 \mathrm{mg}$ iv. IV Ringer Lactate was started. patients received sedation Inj. Midazolam $0.03 \mathrm{mg} / \mathrm{kg}$ IV.Inj. Fentanyl $2 \mathrm{mcg} / \mathrm{kg}$. After preoxygenation,A standardized general anesthetic regime was employed, consisting of propofol $(2 \mathrm{mg} / \mathrm{kg})$ and vecuronium $(0.1 \mathrm{mg} / \mathrm{kg})$, with intraoperative non-opioid analgesia of paracetamol $(15-20 \mathrm{mg} / \mathrm{kg})$ and diclofenac $(0.5 \mathrm{mg} / \mathrm{kg})$.

The anaesthetic gas Nitrous oxide: Oxygen were used in a 2: 1 ratio using the circle circuit and end tidal isoflurane concentration was adjusted between 0.5 and $1.0 \%$.In Group $\mathrm{A}(\mathrm{n}=50)$, ETT cuffs were inflated with Air and in Group W(n=50), ETT cuffs were inflated with distilled water. ETTs made up of polyvinyl chloride were used with high volume low pressure cuffs. ETT Sizes chosen were 7.0 or $7.5 \mathrm{~mm}$ internal diameter (ID) for female patients and 8.0 or $8.5 \mathrm{~mm}$ ID for male patients. All ETTs were checked for leaks 
and the absence of air bubbles ensured in Group W by priming with water at the time of checking. Atraumatic intubations were performed with an oral ETT and its cuff will be inflated with either air(group A) or distilled water(group W) until there was no audible leak. A Pressure Transducer and Pressure Gauge were connected to the ETT pilot ballon at the same time using Three way. Simultaneous Pressure Readings were recorded in the units of $\mathrm{mmHg}$ for both Pressure Gauge and Pressure Transducer during every documentation.

The first pressure reading was measured immidiately after ETT was connected to the circuit.This was taken as " 0 " time and further readings were recorded at 5, 10,15,30, 45,60 minutes and thereafter at 90 minutes and 120 minutes. These pressures were measured simultaneously by Pressure Gauge and Pressure Transducer by attaching the tubing with three way. Complications which were looked for due to excessive cuff pressure are as follows:

1.) Sore throat 2.)Horseness of voice 3.)Others

These complications were assessed after extubating the patients following completion of surgery.

The results were analyzed using Mann-Whitney test, Chi-Square Tests and Wilcoxon Signed Ranks Test.

\section{Results}

There were no significant differences between two groups with respect to age, gender and ASA status.

Table 1: Demographic Data

\begin{tabular}{|l|l|c|c|}
\hline Demographic data & $\begin{array}{l}\text { Group A } \\
(\mathrm{n}=50)\end{array}$ & $\begin{array}{c}\text { Group W } \\
(\mathrm{n}=50)\end{array}$ & P value \\
\hline Age $(\mathrm{yrs})$ & $45.26 \pm 14.16$ & $44.52 \pm 12.53$ & Not Significant \\
\hline Sex $(\mathrm{M} / \mathrm{F})$ & $28 / 22$ & $21 / 29$ & Not Significant \\
\hline
\end{tabular}

Mean cuff pressure in Air and distilled water inflated cuffs are shown in figure $1 \& 2$.

Mean cuff pressure in Group A exceeded those in Group W throughout the study with significant rise in 15 minutes and maximum at the end of 2 hours $(\mathrm{P}<0.05)$

Table 2 Comparison between endotracheal tube cuff pressures $(\mathrm{mmHg})$ values using pressure gauge at various time intervals in group A and group w cases

\begin{tabular}{|c|c|c|c|c|c|c|c|}
\hline Variables $\wedge$ & Group & Mean & SD & Median & IQR & Z-value & p-value \\
\hline \multirow[t]{2}{*}{ Pressure Gauge- 0 minutes } & A & 16.55 & 0.99 & 16.54 & 1.13 & -2.623 & 0.00871 \\
\hline & W & 16.07 & 0.86 & 16.54 & 1.51 & \multicolumn{2}{|c|}{ Difference is significant } \\
\hline \multirow[t]{2}{*}{ Pressure Gauge- 5 minutes } & A & 17.11 & 1.09 & 17.29 & 1.50 & -4.498 & $6.85 \mathrm{E}-06$ \\
\hline & W & 16.24 & 0.81 & 16.54 & 1.51 & \multicolumn{2}{|c|}{ Difference is significant } \\
\hline \multirow[t]{2}{*}{ Pressure Gauge-10 minutes } & A & 18.51 & 1.52 & 18.04 & 2.25 & -6.180 & $6.42 \mathrm{E}-10$ \\
\hline & W & 17.04 & 0.52 & 17.29 & 0.75 & \multicolumn{2}{|c|}{ Difference is significant } \\
\hline \multirow[t]{2}{*}{ Pressure Gauge-15 minutes } & A & 20.27 & 2.11 & 19.92 & 3.76 & -7.329 & $2.31 \mathrm{E}-13$ \\
\hline & W & 17.58 & 0.69 & 17.29 & 0.75 & \multicolumn{2}{|c|}{ Difference is significant } \\
\hline \multirow[t]{2}{*}{ Pressure Gauge-30 minutes } & A & 23.18 & 1.99 & 22.55 & 1.88 & -8.634 & $5.92 \mathrm{E}-18$ \\
\hline & W & 18.37 & 0.90 & 18.04 & 1.50 & \multicolumn{2}{|c|}{ Difference is significant } \\
\hline \multirow[t]{2}{*}{ Pressure Gauge- 45 minutes } & A & 26.21 & 1.98 & 25.56 & 3.00 & -8.735 & $2.43 \mathrm{E}-18$ \\
\hline & W & 19.54 & 0.87 & 19.54 & 0.76 & \multicolumn{2}{|c|}{ Difference is significant } \\
\hline \multirow[t]{2}{*}{ Pressure Gauge-60 minutes } & A & 29.35 & 1.99 & 28.95 & 2.25 & -8.697 & $3.39 \mathrm{E}-18$ \\
\hline & W & 20.84 & 1.01 & 20.30 & 1.50 & \multicolumn{2}{|c|}{ Difference is significant } \\
\hline \multirow[t]{2}{*}{ Pressure Gauge-90 minutes } & A & 34.19 & 1.11 & 34.21 & 0.75 & -8.720 & $2.78 \mathrm{E}-18$ \\
\hline & W & 22.31 & 0.76 & 22.55 & 0.75 & \multicolumn{2}{|c|}{ Difference is significant } \\
\hline \multirow[t]{2}{*}{ Pressure Gauge-120 minutes } & A & 38.16 & 0.93 & 38.34 & 0.75 & -8.739 & $2.35 \mathrm{E}-18$ \\
\hline & W & 24.27 & 1.03 & 24.81 & 1.51 & \multicolumn{2}{|c|}{ Difference is significant } \\
\hline
\end{tabular}

Data failed 'Normality' test. Hence Mann-Whitney test applied. 
Figure 1

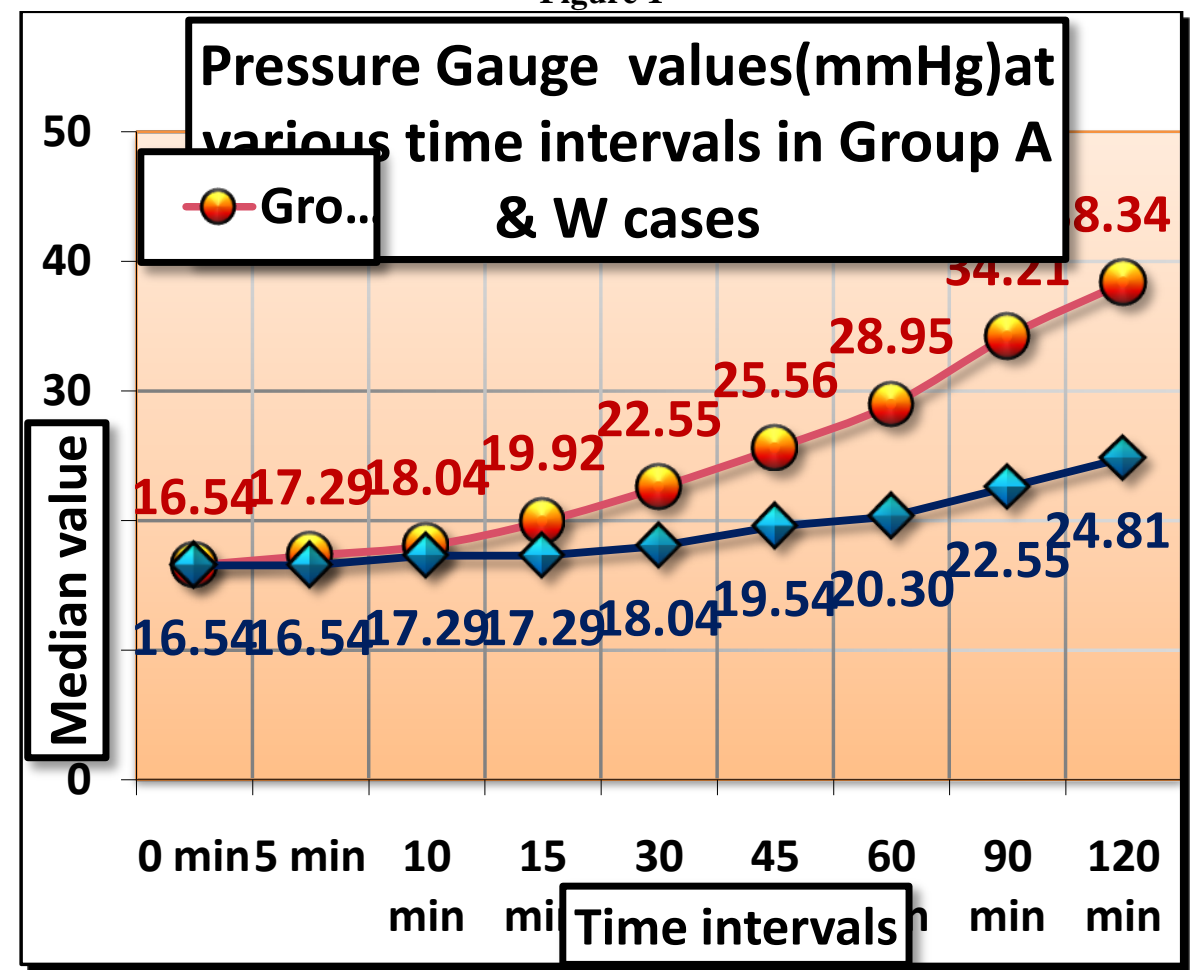

Table 3 Comparison between endotracheal tube cuff pressures $(\mathrm{mmHg})$ values using pressure transducer at various time intervals in group $\mathrm{A}$ and group $\mathrm{W}$ cases

\begin{tabular}{|c|c|c|c|c|c|c|c|}
\hline Variables $\wedge$ & Group & Mean & SD & Median & IQR & Z-value & $\mathrm{p}$-value \\
\hline \multirow[t]{2}{*}{ Pressure Transducer- 0 minutes } & A & 16.26 & 0.97 & 16.00 & 26.25 & -2.623 & 0.00871 \\
\hline & W & 15.76 & 0.69 & 16.00 & 20.75 & \multicolumn{2}{|c|}{ Difference is significant } \\
\hline \multirow[t]{2}{*}{ Pressure Transducer-5 minutes } & A & 16.82 & 1.10 & 17.00 & 1.25 & -4.498 & $6.85 \mathrm{E}-06$ \\
\hline & $\mathrm{W}$ & 15.88 & 0.66 & 16.00 & 1.00 & \multicolumn{2}{|c|}{ Difference is significant } \\
\hline \multirow[t]{2}{*}{ Pressure Transducer-10 minutes } & A & 18.20 & 1.39 & 18.00 & 2.00 & -6.180 & $6.42 \mathrm{E}-10$ \\
\hline & $\mathrm{W}$ & 16.66 & 0.69 & 17.00 & 1.00 & \multicolumn{2}{|c|}{ Difference is significant } \\
\hline \multirow[t]{2}{*}{ Pressure Transducer-15 minutes } & A & 19.86 & 1.93 & 19.50 & 2.00 & -7.329 & $2.31 \mathrm{E}-13$ \\
\hline & W & 17.32 & 0.77 & 17.00 & 1.00 & \multicolumn{2}{|c|}{ Difference is significant } \\
\hline \multirow[t]{2}{*}{ Pressure Transducer-30 minutes } & A & 22.62 & 1.95 & 22.00 & 3.00 & -8.634 & $5.92 \mathrm{E}-18$ \\
\hline & W & 18.14 & 0.78 & 18.00 & 1.00 & \multicolumn{2}{|c|}{ Difference is significant } \\
\hline \multirow[t]{2}{*}{ Pressure Transducer-45 minutes } & A & 25.64 & 1.97 & 25.00 & 2.25 & -8.736 & $2.42 \mathrm{E}-18$ \\
\hline & W & 19.14 & 0.76 & 19.00 & 1.00 & \multicolumn{2}{|c|}{ Difference is significant } \\
\hline \multirow[t]{2}{*}{ Pressure Transducer-60 minutes } & A & 28.78 & 2.01 & 28.50 & 3.00 & -8.697 & $3.39 \mathrm{E}-18$ \\
\hline & W & 20.30 & 0.89 & 20.00 & 1.00 & \multicolumn{2}{|c|}{ Difference is significant } \\
\hline \multirow[t]{2}{*}{ Pressure Transducer-90 minutes } & A & 33.58 & 1.13 & 33.50 & 2.00 & -8.720 & $2.78 \mathrm{E}-18$ \\
\hline & W & 21.74 & 0.88 & 22.00 & 1.00 & \multicolumn{2}{|c|}{ Difference is significant } \\
\hline \multirow[t]{2}{*}{ Pressure Transducer-120 minutes } & A & 37.58 & 0.93 & 38.00 & 1.00 & -8.739 & $2.35 \mathrm{E}-18$ \\
\hline & W & 23.66 & 0.94 & 24.00 & 1.00 & \multicolumn{2}{|c|}{ Difference is significant } \\
\hline
\end{tabular}

$\wedge$ Data failed 'Normality' test. Hence Mann-Whitney test applied. 


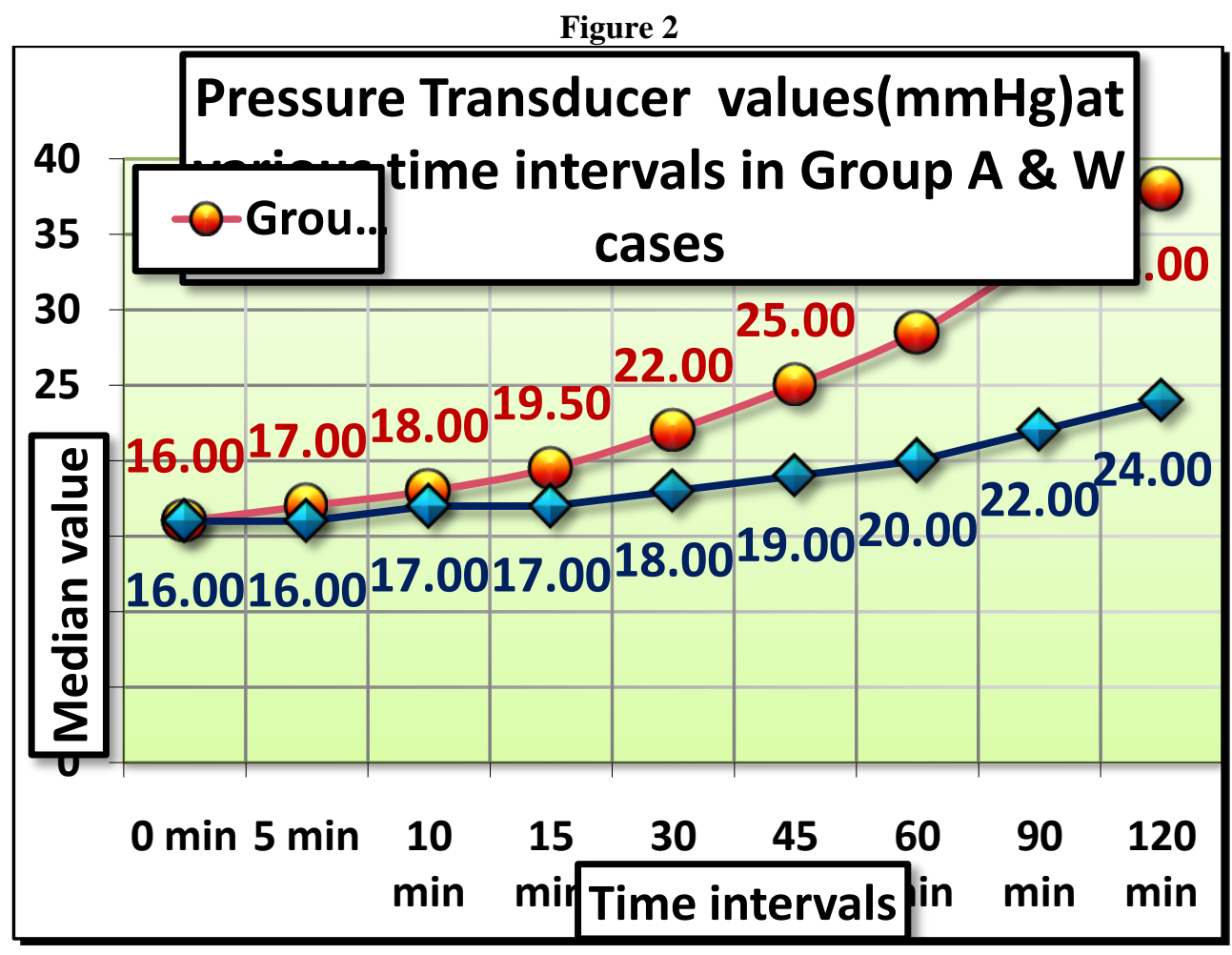

\section{DISCUSSION}

The inflatable cuff on an endotracheal tube should provide both an effective airway seal and protection from aspiration, without causing significant trauma to tracheal mucosa. However, with time, the pressure inside cuff of standard endotracheal tube is seen to rise in the setting of Nitrous Oxide - Oxygen Anesthesia. This has serious practical implications on the patient, as this pressure is likely to be exerted on the lateral walls of trachea. This pressure when exerted by use of an endotracheal tube cuff on the walls of trachea where the first contact is made with the tracheal wall then transmits this pressure to that part of trachea. This results in mucosal damage, cartilaginous necrosis and eventual tracheal stenosis. All these complications have been reported in many scientific papers till date, when endotracheal tubes were used for a longer duration of time. In our study we found that final ETT cuff pressures in Air filled cuffs at the end of 2 hours was $38.16 \pm 0.93 \mathrm{mmHg}$ with pressure gauge and $37.58 \pm 0.93 \mathrm{mmHg}$ with pressure transducer.Final pressures in distilled water filled cuffs at the end of 2 hours was $24.27 \pm 1.03 \mathrm{mmHg}$ with pressure gauge and $23.66 \pm 0.94 \mathrm{mmHg}$ with pressure transducer.

In Both groups values of pressures measured by pressure gauge and pressure transducer at each measurement are different. This difference is statistically significant as $\mathrm{P}$ value is $<0.05$. This difference does not seem clinically significant. Measurement with Pressure Transducer provides one single numerical value.

On the other hand, measurement with Pressure gauge provides numerical value in a range, which is liable to incorrect interpretation because of observer error. These values roughly corresponds to the range which falls in standard deviation for values of pressure transducer at each point of measurement. So Pressure gauge or pressure transducer both can be used to measure ETT cuff pressure, however values obtained by pressure transducer are more precise. In this study, Pressure started rising after 15 minutes in Group A and this rise is very obvious from 30 minutes onwards. The difference in ETT pressures between Group A and Group W is maximum at 120 minutes.

\section{Conclusion}

In this study we compared endotracheal tube cuff pressure in air and distilled water filled cuffs during general anaesthesia using nitrous oxide.Cuff pressure values obtained by pressure gauge and pressure transducer is also compared.In this study we concluded that- Rise in pressure with time is more in Air filled ETT cuffs as compared to distilled water filled cuffs.ETT cuff pressure values obtained by pressure gauge and pressure transducer are almost equal, so both are equally good for pressure monitoring. Values obtained by pressure transducer are more precise. This study showed no or minimal complications with both groups. 


\section{References}

[1]. Ahmad N.L., Norsidah A.M. Change in endotracheal tube cuff pressure during nitrous oxide anaesthesia: a comparison between air and distilled water cuff inflation. Anaesthesia Intensive care. 2001 Oct;29(5):510-4.

[2]. Patel R.I., Oh T.H. and Epstein B.S. Effects of Nitrous Oxide on Pressure changes of Tracheal tube cuffs following inflation with Air and Saline. Anaesthesia. 1984;39:862-864.

[3]. Chandler M. Pressure changes in tracheal tube cuffs. Anaesthesia.1986 Mar;41(3):287-293.

[4]. Caroll Robert, Hedden Michael, Safar Peter. Intratracheal Cuffs: Performance characteristics. Anaesthesiology. 1969 Sep;31(3):275-81.

[5]. Lin Y, Lin D, Chen B, Ji C, Yuan C, Wang B. Experimental study of the monitoring bias of pressure in intubation balloon using handheld pressure gauge. Zhonghua Wei Zhong Bing Ji Jiu Yi Xue. 2014 May;26(5):347-50. 